\title{
Design and Control of an Active Powered Knee Prosthesis System using Position-based Impedance Control
}

\author{
Bongeon Jo, Young-Sam Lee \\ Inha University \\ Inharo 100, Incheon, Korea \\ jbw0803@gmail.com; lys@inha.ac.kr
}

\begin{abstract}
In this paper, we deal with the implementation of an active powered knee prosthesis using the position-based impedance control. We propose a new mechanical structure for the knee prosthesis such that convenience of the maintenance is much improved and the noise seen in the load cell measurement, caused by a motor, is greatly removed. We develop the embedded system based on a 32-bit digital signal controller and make the standalone operation without tethering possible by attaching the developed embedded system on the prosthesis. We divide the walking cycle into 5 stages and control the prosthesis such that it has different mechanical impedance depending on the stages. For efficient control of the impedance, we firstly derive the relationship between the knee angle and the torque applied on the knee and then propose the position-based impedance controller for the knee prosthesis. We evaluate the walking performance of the knee prosthesis through experiments including level walking and inclined walking. We also analyze the features of the proposed knee prosthesis by comparing the walking data of the proposed prosthesis with normal walking data.
\end{abstract}

Keywords: powered knee prosthesis, position-based impedance control

\section{Introduction}

The number of amputees due to war, disease, and accidents is increasing. Patients who have undergone amputation surgery below knee have a knee joint, so walking with a prosthesis is relatively easy [1]. However, in femoral amputees, an unnatural gait occurs when a passive prosthesis is worn. Various types of prosthesis have been developed for the natural walking movement of these amputees.

The developed prosthesis can be divided into passive prosthesis and active prosthesis. Passive prosthesis consists of a structure that stores energy and reduce the physical burden of the wearer [2]. However, since it uses the power of the wearer to walk, amputees consume about $60 \%$ more energy than normal person [3]. A passive prosthesis with a power source adjusts the impedance to assist walking [4], [5], [6], [7]. The active prosthesis controls the knee joint angle, so it has less physical burden than the passive prosthesis, and allows natural walking. Previous research groups have been studying the active prosthesis of various structures and the active prosthesis that can walk in a complex walking environment [8], [9].

Impedance control used in industrial robots or wearable robots allows the robots to adapt to uncertain environments [10]. The active powered knee prosthesis aims at producing a movement similar to that of a normal person. The powered prosthesis uses impedance control to cope with the wearer's movement and external load. Imitating movement at the same level as the human body can be linked to robot technology such as humanoid [11].

The paper is structured as follows: In Section 2, we describe the design of the active powered knee prosthesis. In Section 3, we describe how to divide the gait phase and implement a position-based impedance controller. In Section 4, we perform experiments using the powered knee prosthesis and present the results. Finally, in Section 5, we make conclusions

\section{Design of an active powered knee prosthesis}

\subsection{Mechanical design}

We design a powered knee prosthesis that removes the drawbacks of previous studies [12]. It has a slider crank structure that uses a DC motor and a ball screw for actuation as shown in Figure 1-(a), where $x$ is the actuator's length, $l_{1}$ and $l_{2}$ are the distance between the axles, $\alpha_{0}$ is the initial angle when the knee angle is $0^{\circ}$, and $\theta$ is the knee angle. The 
magnetic encoder for measuring the knee angle is in the position $\Phi$ and the load cell for measuring the load of the knee joint is in the position (2). (3) and (4) are a DC motor and a ball screw which are used as an actuator of the powered knee prosthesis respectively. The DC motor is equipped with an incremental encoder. Consequently, it can be used for speed control or position control.

(a)

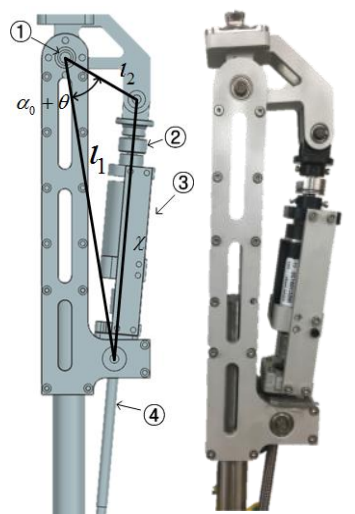

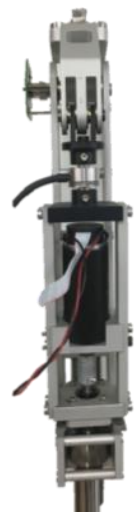

(b)

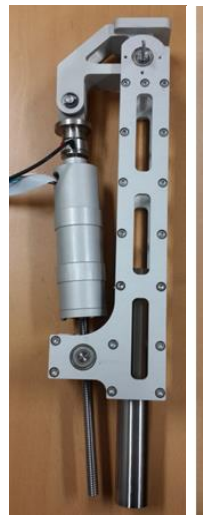

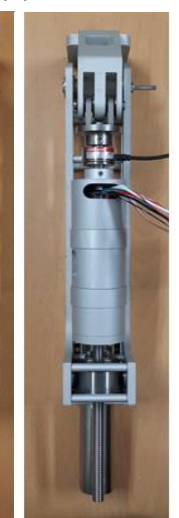

Fig. 1: Mechanical structure: (a) proposed powered knee prosthesis, (b) existing powered knee prosthesis.

Figure 1-(b) shows the powered knee prosthesis's actuator used in the previous study [12]. Since it has a cylindrical structure, it is not easy to replace when it needs to be replaced for reasons such as breakdown or change of parts. In addition, the two parts that fix the load cell are made of aluminium. Since the load cell is located close to a DC motor through aluminium parts, the load cell signal is easily affected by the motor switching noise. There are two differences when compared to the existing prosthesis presented in [12]. First, the actuator is designed to be fixed using two side supports. This has the advantage that components such as DC motor, ball screw, and coupling can be easily replaced. Second, the parts that fix the load cell are made of nylon. Using nylon, the insulation material, can prevent the noise generated by the motor from affecting the load cell signal through the prosthesis's frame.

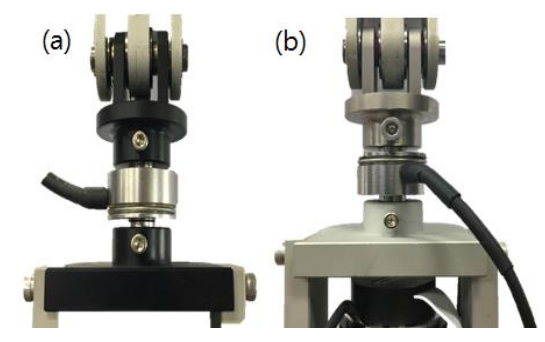

Fig. 2: Assembling the load cell: (a) using nylon parts, (b) using aluminium parts.

Figure 2 shows that the load cells are fixed using nylon parts and aluminium parts, respectively. The following experiment is conducted to confirm the difference between the two methods. We fix the prosthesis to the apparatus for the experiment and apply the sine wave voltage input to the DC motor. PWM for the DC motor drive uses 20Khz carrier frequency and synchronizes the time when the underflow of PWM internal counter occurs and the time when the ADC is done. The sampling time is $2 \mathrm{~ms}$ and 40 data are measured per sampling time. The 40 data are processed with an average filter. A lab-built data acquisition system is used for data measurement [13].

The existing powered knee prosthesis uses aluminium parts to fix the load cell [14]. Figure 3-(a) shows the force data measured when using nylon parts and figure 3-(b) is the force data measured when using aluminium parts. The force value increases when the knee is bent and the force value decreases when the knee is flexed. When using nylon parts, the noise size is about 5 ADC units, which is evenly distributed during flexion and extension. When aluminium parts are used, the influence of noise becomes large when the knee is flexed. When the knee is flexed, more current flows in the DC motor. 
The noise size is about $25 \mathrm{ADC}$ units, which is five times larger than that in the case of using nylon parts. It is seen that the influence of the noise increases when the sign of the voltage applied to the DC motor is changed. This value is also five times larger than that for nylon parts.
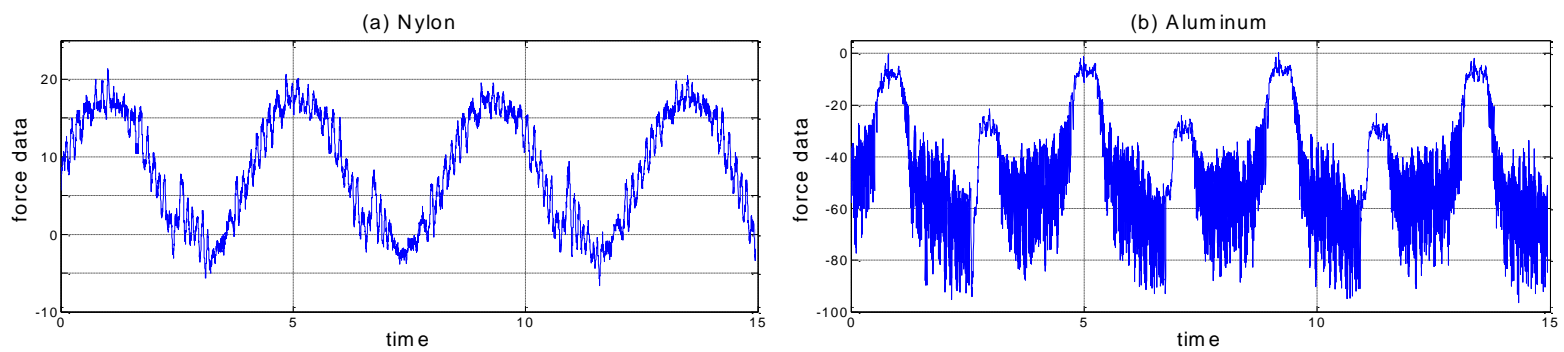

Fig. 3: The signals of the load cell depending on the material of the parts: (a) nylon, (b) aluminium.

By the principle of virtual works, the relationship of the force generated by the actuator and the torque of the knee joint is described, according to [15], as follows:.

$$
\tau_{\mathrm{k}}=F \frac{\partial x}{\partial \theta}
$$

where $\tau_{\mathrm{k}}$ is the torque of knee joint and $F$ is the force generated by the actuator. The force generated by the actuator can be expressed as follows:

$$
\mathrm{F}=\frac{2 \pi}{\mathrm{L}} \tau_{\mathrm{m}}
$$

where $\mathrm{L}$ is the lead of ball screw and $\tau_{\mathrm{m}}$ is the torque of DC motor. The actuator length can be geometrically expressed in the following form:

$$
x=\sqrt{l_{1}^{2}+l_{2}^{2}-2 l_{1} l_{2} \cos \left(\alpha_{0}+\theta\right)} .
$$

Substituting Eqs. (2) and Eqs. (3) into Eqs. (1) yields the following result:

$$
\tau_{\mathrm{k}}=\frac{2 \pi}{\mathrm{L}} \frac{l_{1} l_{2} \sin \left(\alpha_{0}+\theta\right)}{\sqrt{\mathrm{l}_{1}^{2}+l_{2}^{2}-2 l_{1} l_{2} \cos \left(\alpha_{0}+\theta\right)}} \tau_{\mathrm{m}}=\mathrm{g}(\theta) \tau_{\mathrm{m}},
$$

where $g(\theta)$ is a function of the relationship between the motor torque and the knee joint torque, as shown in Figure 4.

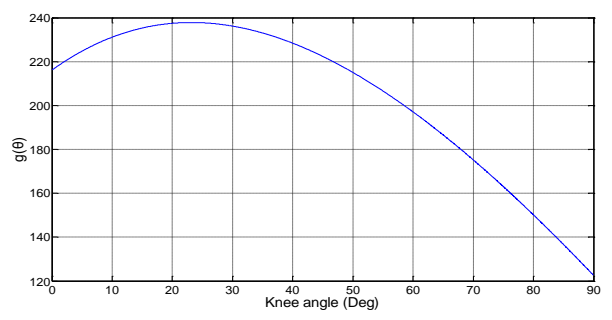

Fig. 4: Relationship between motor torque and knee joint torque. 
The motor used in this paper is RE40 of MAXON company with $150 \mathrm{~W}$ output rating. The rated voltage is $48 \mathrm{~V}$, the no-load speed is $7590 \mathrm{rpm}$, and the torque constant is $60.3 \mathrm{mNm} / \mathrm{A}$. The allowable current of the motor driver used in this paper is $10 \mathrm{~A}$. Therefore, the maximum knee joint torque is $143.4 \mathrm{Nm}$ in $23^{\circ}$. In the case of a person weighing $75 \mathrm{~kg}$, the knee joint torque required for a level walking is $43 \mathrm{Nm}$, so the powered knee prosthesis designed in this paper is suitable for level walking.

\subsection{Embedded system design}

The embedded system designed for the control of the powered knee prosthesis consists of a board for sensor signal processing and control operation and a DC motor driver board. Figure 5 shows the configuration of the embedded system. As a main microcontroller, we use the TMS320F2811 of TI company. The following system components are used for powered knee prosthesis control. A magnetic encoder for measuring the knee joint angle, a load cell for measuring the force applied to the knee joint, an FSR sensor for discriminating the phase of gait, a DC motor driver for driving a DC motor, an incremental encoder for position control, and monitoring system to check the internal data of the microcontroller. The method of interfacing each component with the microcontroller is shown in Figure 5. The control board and the DC motor driver are attached to the frame of the powered knee prosthesis and operate independently using the battery.

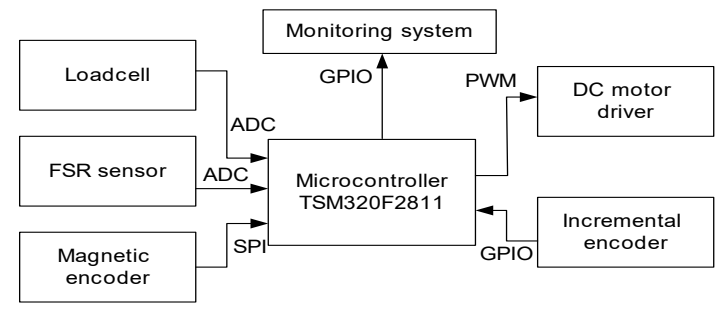

Fig. 5: Embedded system framework.

\section{Control of an active powered knee prosthesis}

\subsection{Gait phase classification}

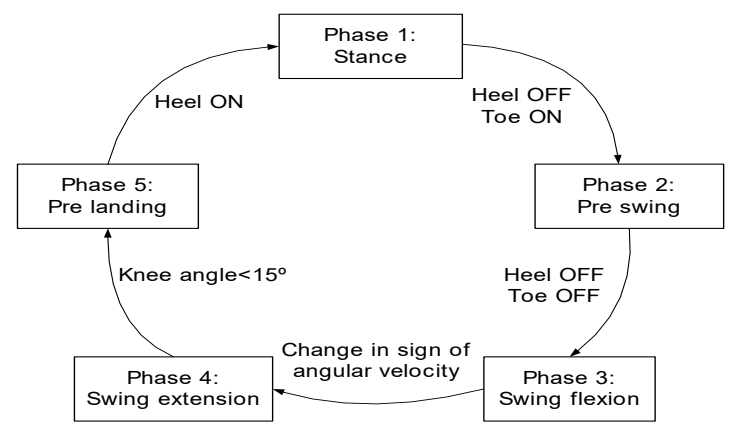

Fig. 6: FSM for gait phase classification.

The gait cycle of a normal person can be divided into a stance phase and a swing phase [16]. The stance phase is a state in which the foot is touching the ground, and the knee joint has high impedance and acts as a buffer against the impact of the foot on the ground. The swing phase is a state in which the foot is separated from the ground, the knee joint has low impedance and the foot is naturally shaken.

In this paper, we divide the gait cycle into five phases. Figure 6 illustrates the FSM(Finite State Machine), which represents the five phase gait cycle. Stance phase (Phase 1) is the period from the moment when the heel touches the ground to the moment when it is separated from the ground. In this phase, the knee joint impedance should be large enough to absorb the shock. Pre swing phase (Phase 2) represents the period from the moment right after the stance phase to the moment when the foot comes off the floor. Swing flexion phase (Phase 3) is from the moment when the foot comes off the 
ground and the flexion of the knee joint occurs to the moment before the extension of the knee occurs. The knee joint has very small impedance and moves by thigh movement. Swing extension phase (Phase 4) is the period until the bent knee is fully extended. As in the previous phase, the knee joint has very small impedance and movement is dependent on thigh movement. Finally, pre landing phase (Phase 5) is the period until the fully extended knee reaches the ground. The knee joint has very high impedance.

\subsection{Control of an active powered prosthesis}

The powered knee prosthesis should move the knee joint appropriately according to the wearer's gait cycle. When walking, the powered knee prosthesis is affected by external factors such as the walking environment and the walking speed. Therefore, it is impossible to imitate the walking of a normal person through the control of the knee joint angle. In this paper, we use the impedance control instead of the position control to create the motion that responds to the load on the knee.

The knee torque is described in [3] by a spring and damper system, as follows:

$$
-\tau_{\mathrm{k}}(t)=k_{\mathrm{i}}\left(\theta_{\mathrm{k}}(t)-\theta_{\mathrm{k}, i, e q}\right)+b_{i} \dot{\theta_{\mathrm{k}}}(t)
$$

where $k$ is the spring coefficient, $b$ is the damper coefficient, $\theta_{\mathrm{k} \text {,eq }}$ is the equilibrium point of the knee angle, and the subscript $i$ is the gait phase. Since the knee joint impedance should be different for each gait phase, a damper model and a spring-damper model are selected and used. Considering the powered knee prosthesis as a damper model, the spring coefficient can be removed and the knee joint angle can be regarded as reference of a control. The knee joint angle can be obtained as shown in Eqs. (6), and transformed into a discrete Eqs. (7).

$$
\begin{gathered}
\theta_{\mathrm{k}}(t)=-\int_{0}^{t} \frac{\tau_{\mathrm{k}}(s)}{b_{i}} d s . \\
\theta_{\mathrm{k}}[n+1]=\theta[n]-\frac{\tau_{\mathrm{k}}}{b} \times d t .
\end{gathered}
$$

Considering the powered knee prosthesis as a spring-damper model, the knee joint angle, which is the reference of a control, is expressed as:

$$
\theta_{\mathrm{k}}(t)=\left(\theta_{\mathrm{k}, i, 0}-\theta_{\mathrm{k}, i, e q}+\frac{\tau_{\mathrm{k}}(t)}{k_{i}}\right) \exp \left(-\frac{k_{i}}{b_{i}} \times t\right)+\theta_{\mathrm{k}, i, e q}-\frac{\tau_{\mathrm{k}}(t)}{k_{i}}
$$

where $\theta_{\mathrm{k}, i, 0}$ is the knee angle when the gait phase changes.

$\theta_{\mathrm{k}}(t)$ obtained from Eqs. (6) or Eqs. (8) produces the knee joint impedance as Eqs. (5). By using the Eqs. (7) or Eqs. (8) to generate the angle reference input of the knee joint and controlling the position of the knee joint to follow the reference input though the position control, the knee joint impedance is controlled approximately as the Eqs. (5).

In this paper, we used the position-based impedance control. The method of measuring the knee joint angle is a direct method using a magnetic encoder and an indirect method using an incremental encoder and Eqs. (3). We calculate the knee joint angle using an indirect method and use it for position control.

The initial value of the incremental encoder is unknown. Therefore, the initial knee joint angle value, $\theta_{\mathrm{k}, 0}$, is measured by using a magnetic encoder when the system is started. The initial actuator length can be obtained by using $\theta_{\mathrm{k}, 0}$ and Eqs. (3). The reference actuator length is calculated by using the reference knee angle and Eqs. (3). The current actuator length is calculated by using the rotational displacement of the motor and the lead length of the ball screw and expressed as follows:

$$
x=x_{0}+L \times \theta_{\mathrm{m}}
$$

where $x_{0}$ is the initial actuator length. The reference rotational displacement is calculated by dividing the lead length of the ball screw by the difference between the current actuator length and the reference actuator length and expressed as follows: 


$$
\theta_{\mathrm{m}, \mathrm{ref}}=\frac{x_{r e f}-x}{L}
$$

where $x_{r e f}$ is the reference actuator length and $\theta_{\mathrm{m}, \mathrm{ref}}$ is the reference rotational displacement of the DC motor.

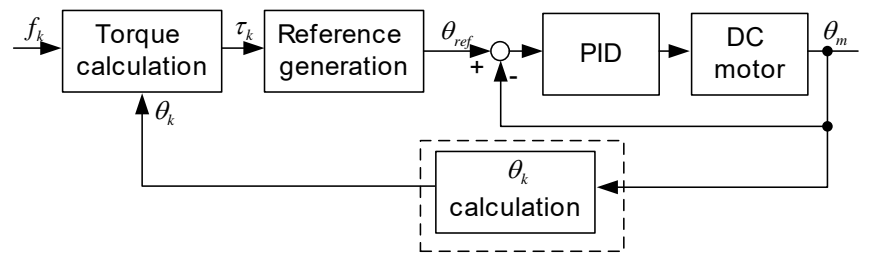

Fig. 7: Block diagram for control of powered knee prosthesis.

Figure 7 represents the block diagram for control of the powered knee prosthesis. In the dotted block, the knee joint angle is calculated using Eqs. (11), which is the modification of Eqs. (3)

$$
\theta_{\mathrm{k}}=\cos ^{-1}\left(\frac{l_{1}^{2}+l_{2}^{2}-x^{2}}{2 l_{1} l_{2}}\right)-\alpha_{0}
$$

\section{Experiments and results}

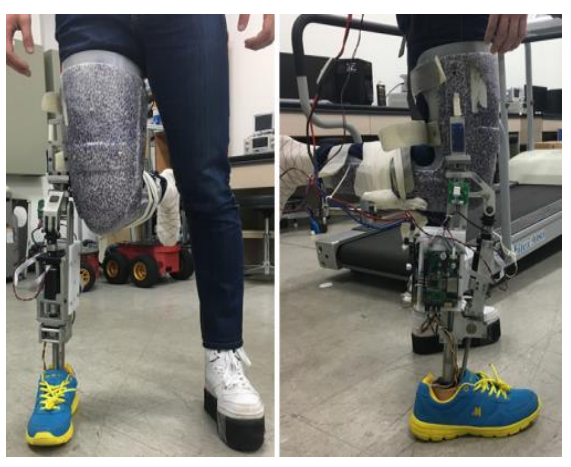

Fig. 8: Wearing a powered knee prosthesis using an adapter.

Figure 8 shows a normal person wearing the powered knee prosthesis using an adapter for the experiments. We collect the data using a lab-built DAQ system. Using a treadmill, we experiment the level walking and inclined walking. We use the same impedance controller in experiments for level walking and inclined walking. Table 1 presents the coefficients. The coefficients were selected by trials and errors so that the wearer can feel natural walking.

Table 1: Impedance controller models and coefficients.

\begin{tabular}{|c|c|c|c|c|}
\hline Phase & Model & $k$ & $b$ & $\theta_{\mathrm{eq}}$ \\
\hline 1 & Spring-damper & 5 & 0.1 & 15 \\
\hline 2 & Spring-damper & 1 & 0.05 & 25 \\
\hline 3 & Spring-damper & 0.3 & 0.01 & 65 \\
\hline 4 & Damper & - & 0.007 & - \\
\hline 5 & Position control & - & - & - \\
\hline
\end{tabular}




\subsection{Level walking and inclined walking}

Figure 9-(a) shows the average knee joint angle during level walking of normal person. Two knee flexion and extension occur at the knee joint when level walking. Figure 9-(b) and Figure 9-(c) show the knee joint angle when level walking at speeds of $2.5 \mathrm{~km} / \mathrm{h}$ and $1.5 \mathrm{~km} / \mathrm{h}$, respectively. The two flexion angles of the knee joint at the speed of $2.5 \mathrm{~km} /$ $h$ are $16^{\circ}$ and $45^{\circ}$ on average. The two flexion angles of the knee joint at the speed of $1.5 \mathrm{~km} / \mathrm{h}$ are $17^{\circ}$ and $47^{\circ}$ on average.

(a)

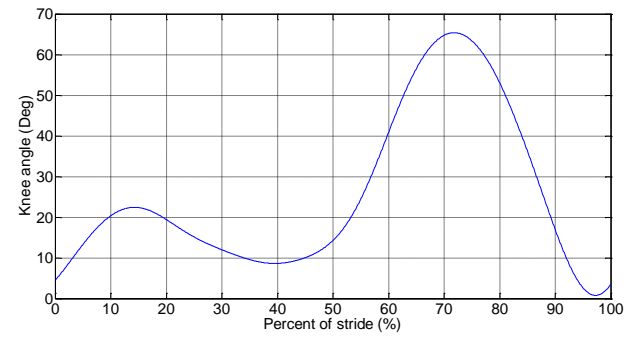

(b)

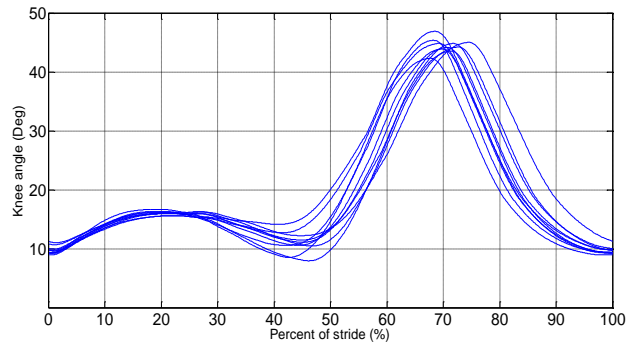

(c)

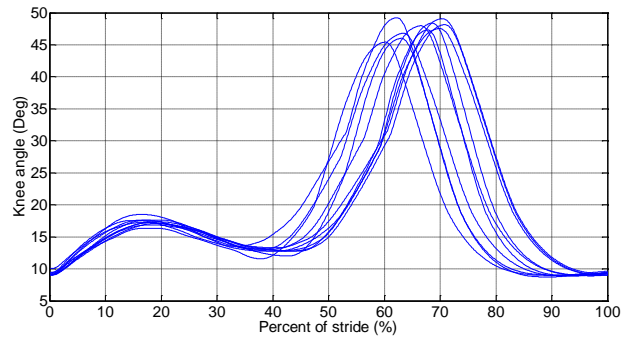

Fig. 9: Knee angle during level walking: (a) $2.5 \mathrm{~km} / \mathrm{h}$ with prosthesis, (b) $1.5 \mathrm{~km} / \mathrm{h}$ with prosthesis (c) normal walking.

Figure 10 shows the angle of the knee joint when inclined walking at 7 degree slope. In general, as the inclination angle increases, the fist first flexion angle becomes larger, and the later flexion angle becomes smaller [17]. The two flexion angles of the knee joint at 7 degree slope are $16^{\circ}$ and $42^{\circ}$ on average

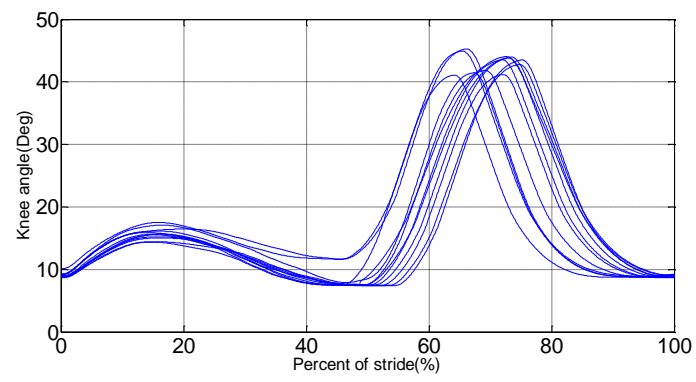

Fig. 10: Knee angle during inclined walking.

\subsection{Experiment result}

The results show that the powered knee prosthesis operates according to the walking speed because of impedance control. The first flexion in level walking appears in the stance phase. The difference between normal walking and the powered knee prosthesis is about $5^{\circ}$. The second flexion occurs when the phase changes from swing flexion to swing extension. The difference between normal walking and the powered knee prosthesis is about $18^{\circ}$. In addition, it is irregular when the second flexion occurs at a slow speed. This is caused by wearing the powered knee prosthesis using the adapter, and thus it is difficult to balance during walking because the powered knee prosthesis are next to the original position. The subject moves the opposite leg early to avoid falling. Since the powered knee prosthesis uses impedance control, it operates irregularly. If we adjust the coefficient to make the second flexion angle similar to the normal case, the knee joint will have high impedance. As a result, the powered prosthesis operates with a determined trajectory. When inclined walking, the first flexion angle appears to be similar to that of the level walking, because the one degree of freedom powered knee prosthesis does not have the ankle.

\section{Conclusion}

In this paper, we designed the active powered knee prosthesis and divided the gait cycle into several stages for control. We also designed the position-based impedance controller and performed experiments. The proposed powered prosthesis was developed such that it can remove the existing drawbacks. The actuator was designed to be fixed using two side 
supports so that maintenance is easy. In addition, the load cell was fixed with nylon mechanical parts. Therefore, the noise of the load cell signal was much reduced. In order to control the active powered knee prosthesis, the gait cycle was divided into 5 phases and the FSM was constructed accordingly. The position-based impedance control was constructed to control the impedance of the prosthesis. We considered the powered knee prosthesis as a mechanical system with a spring and damper and controlled the knee joint angle to generate the impedance for each phase. We analyzeqd the difference between walking using a powered knee prosthesis and normal walking through the experiment of level walking and inclined walking.

\section{Acknowledgements}

This research was supported by Korea Electric Power Corporation through Korea Electrical Engineering \& Science Research Institute. (Grant number: R15XA03-12)

\section{References}

[1] World Health Organization United States Departmentof Defense MossRehab Amputee Rehabilitation Program MossRehab Hospital, A Manual forthe Rehabilitation of People with Limb Amputation, USA, 2004.

[2] K. H. Kim, Y. H. Kim, S. K. Kim, G. T. Yang, and M. S. Mum, "Development of transfemoral prostheses and its gait analysis," in Proc. of the Conf. KSME 1996, vol. 2, no. 1, pp. 303-308, 1996.

[3] F. Sup, H. A. Varol, J. Mitchell, T.J. Withrow and M. Goldfarb, "Preliminary Evaluations of a Self-Contained Anthropomorphic Transfemoral Prosthesis," IEEE/ASME Transactions on mechatronics, vol. 14, no. 6, pp. 667-676, 2009.

[4] B. G. A. Lambrecht and H. Kazerooni, "Design of a Semi-Active Knee Prosthesis," in Proc. of the Conf. IEEEInternational Conference on Robotics and Automation, Kobe, Japan, pp. 639-645, 2009.

[5] J. P. Park, J.W. Kang and S. B. Choi, "Design and Analysis of Above Knee Prosthetic Leg Using MR Damper," Transactions of the Korean Society for Noise and Vibration Engineering, vol. 26, no. 2, pp. 165-171, 2016.

[6] F. Li, H. Xie, W. Yuan ancd Y. Liu, "The Application Research of MR Damper in Intelligent Bionic Leg," Chinese Control and Decision Conference, Guilin, China, pp. 1327-1331, 2009.

[7] H. Herr and A. Wilkenfeld, "User-adaptive controlof a magnetorheological prosthetic knee," Industrial Robot: An International Journal, vol. 30, no. 1, pp. 42-55, 2003.

[8] A. H. Shultz, B. E. Lawson and M. Goldfarb, "Running With a Powered Knee and Ankle Prosthesis," IEEE Transactions on Neural Systems and Rehabilitation Engineering, vol. 23, no. 3, pp. 403-412, 2015.

[9] S. Au, M. Berniker and H. Herr, "Powered ankle-foot prosthesis to assist level-ground and stair-descent gait," Neural Networks, vol. 21, no. 4, pp. 654-666, 2008.

[10] J. K. Ahn and S. Jung, "Position-Based Force Control Application of a Mobile Robot with Two Arms," Journal of Institute of Control, Robotics and Systems, vol. 19, no. 4, pp. 315-321, 2013.

[11] J. H. Park, "Impedance Control for Biped Robot Locomotion," IEEE TRANSACTIONS ON ROBOTICS AND AUTOMATION, vol. 17, no. 6, 2001.

[12] G. Y. Gyeong, J. G. Kim and Y. S. Lee, "The Structure of a Powered Knee Prosthesis based on a BLDC Motor and Impedance Control using Torque Estimation on Free Swing," Journal of Institute of Control, Robotics and Systems, vol. 21, no. 5, pp. 407-412, 2015.

[13] S. K. Seo, Y. S. Park and Y. S. Lee, "Development of a Cost-effective Data Acquisition System using an Opensource Hardware and Matlab/Simulink," in Proceedings of ICINCO 2016, Lisbon, Portugal, pp. 484-491, 2016.

[14] W. S. Kim, S. Y. Kim and Y. S. Lee, "Development of a Powered Knee Prosthesis using a DC Motor," Journal of Institute of Control, Robotics and Systems, vol. 20, no. 2, pp. 193-199, 2014.

[15] C. D. Hoover, G. D. Fulk and K. B. Fite, "The Design and Initial Experimental Validation of an Active Myoelectric Transfemoral Prosthesis," Journal of Medical Devices, vol. 6, no. 1, 2012.

[16] M. W. Whittle, Gait Analysis an Intoroduction, $4^{\text {th }}$ Ed, Elsevier, 2007.

[17] N. S. Yun, K. O. Lee and J. Y. Kim, "Kinematic comparison of walking on various inclined walkways," Journal of Korean Association of Physical Education and Sport for Girls and Women, vol. 13, no. 1, pp. 89-101, 1999. 